Aus dem Sanatorium Groedel in Bad Nauheim.

\title{
Antisyphilitische Kuren mit intravenöser Darreichung von Salvarsan-Novasurolmischung bei Herzkranken.
}

Von Dr. Carl Winkler, Hausarzt des Sanatoriums.

In neuester Zeit wird das Novasurol - ein in der Syphilisbehandlung bereits bewährtes, intravenös verwendbares Quecksilberpräparat - auch als Diuretikum für die Behandlung hydropischer Herzkranker (La ng e, Ther. d. Gegenw. 1920 Nr. 7 ; KolIert ebenda $1920 \mathrm{Nr}$. 10) verwandt: Wir selbst haben bisher nur bei den schwersten Fällen, und nur wenn alle anderen Mittel versagt hatten, 1,0 Novasurol verabfolgt. Der Erfolg war stets eklatant. Es setzte eine Haruflut ein, dann versagte jedoch die Diurese vollkommen. Gaben wir nun am nächsten Tag wieder Novasurol, so hatten wir keinen Erfolg. Im Gegenteil erlebten wir dann 'schwere Intoxikationserscheinungen, Enteritiden besonders, und einmal ein schweres Quecksilberexanthem. Wir sind deshalb bei schwerer Störung der Diurese etwas zurück haltend geworden. Bei leichteren und auf andere Mittel reagierenden Fällen haben wir noch keine Erfahrungen gesammelt.

Die Tatsache, daß Novasurol bei Kreislaufstörungen günstig auf die Diurese wirkt und von seiten der Zirkulationsorgane gut vertragen wird, legte uns aber den Gedanken nahe, bei unseren zahlreichen tertiärsyphilitischen schweren Herzfällen die einzeitige intravenöse Neosalvarsan-Novasurol-Behandlung durchzuführen, um so mehr, als die vorliegenden Berichte (S ch önfeld, M. m.W. 1921 Nr. 7; daselbst vorhergehende Literatur; Sch malz; D. m.W. 1921 Nr. 35; Treitel, Ther. d. Gegenw., Mai 1921), die sich allerdings vorzugsweise auf frische Fälle bezogen, günstig lauteten.

In Folgendem will ich eine Zusammenstellung der von uns im Jahre 1921 mit intravenöser Darreichung von Salvarsan-Novasurol-

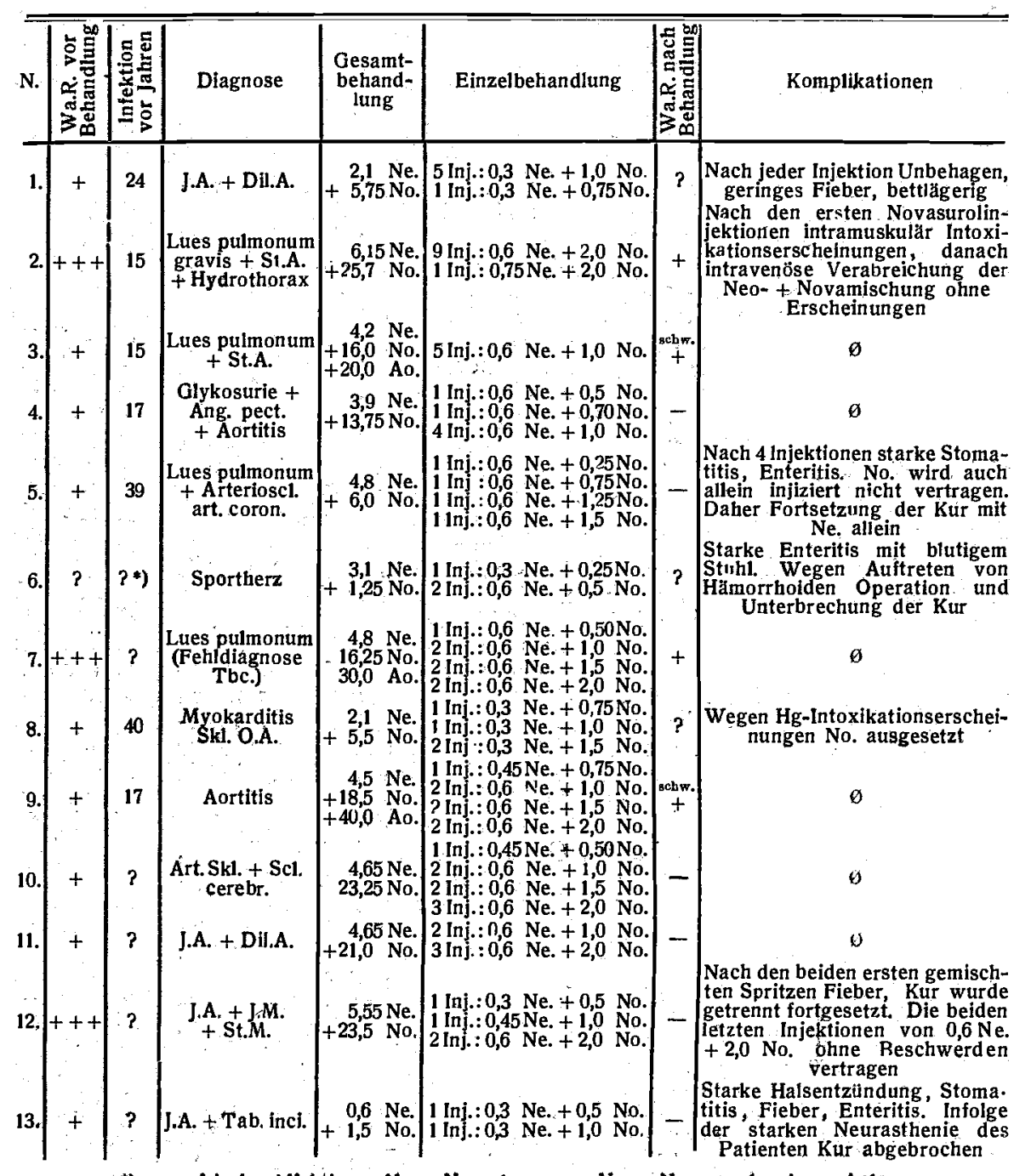

7 ganz frische Affektion. Ne $=$ Neosalvarsan. No. $=$ Novastrol. Ao. $=$ Aolan. 
Mischung behandelten Syphilisfälle bringen und über uthsere Erfahrung berichten. Es handelt sich um 13 Fälle, über welche dié Tabelle Auskunft gibt:

Aus dieser Aufstellung ist $\mathrm{zu}$ ersehen, daB die Verabreichung von Neosalvarsan-Novasurol ' bei 6 Fällen ohne Komplikationen vertragen wurde. $\mathrm{DaB}$ die Intoxikationserscheinungen der übrigen $7 \mathrm{Fälle}$, Enteritis, Stomatitis usw., hauptsächlich auf Novasurol zurückzuführen sind, steht wohl auBer Zweifel. Und es ist zu hoffen, daß man bei vorsichtiger, langsam steigender Dosierung die Mehrzahl der F̈̈lle einzeitig mit Novasurol-Neosalvarsan behandeln kann. Aber eine gewisse Zahl von Menschen wird Novasurol auch bei vorsichtiger Steigerung nicht vertragen, eben wegen seiner starken Wirkung, die offenbar leicht $\mathrm{zu}$ Quecksilberintoxikationserscheinungen führt. Von seiten des Zirkulationsapparates wird jedenfalls Novasurol allein tind in Kombination mit Salvarsan gut vertragen - auch von seiten der Niere sahen wir keine unangenehmen Reaktionen - und gerade bei manchen, therapeutisch besonders schwer zugänglichen Fällen von tertiärer Herz- und Lungensyphilis schien uns nur die kombinierte intravenöse Quecksilber-Salvarsan-Behandlung den subjektiv und objektiv auffallend guten Erfolg hervorzurufen.

Einige Geschicklichkeit setzt allerdings die Technik voraus, da bei

dem undurchsichtigen, dunkelgrünen Gemisch das Eindringen des Venenblutes in die Spritze nicht leicht beobachtet werden kann. Nicht unerwähnt möchte ich lassen, daB auch die intravenöse Verabreichung von Novasurol allein der intramuskulären in jeder Beziehung vorzuziehen ist. Und zwar ist sie, richtig ausgeführt, vọ̈llig schmerzlos und die Wirkung natürlich viel kräftiger. Anderseits ist zuzugeben, daß sich bei längerem Stehen die dunkelgrüne Mischung des Novasurol-Neosalvarsans in eine dunkelgraue verändert. $\mathrm{Ob}$ es sich dabei um eine Aenderung der chemischen Verbindung handelt oder um ein Sedimentieren, entzieht sich meirier Kenntnis. Jedenfalls hätte ich ohne die vorliegenden Mitteilungen nicht gewagt, ein derartiges Gemisch $z \mathfrak{u}$ injizieren.

Vielleicht gelingt es, das Novasurol so darzustellen, daß die Mischung der Lösungen beider Stoffe klar und beständig bleibt. Anderseits könnte man den Uebelstand vielleicht durch Verbesserung der Technik beseitigen, wenn man aus einer Doppelspritze beide Medikamente durch eine Kaniüle in einer Sitzung, aber nacheinander verabreicht. 